\title{
Assessment of blood glucose variability by continuous monitoring as a therapy guide for patients with diabetic nephropathy on hemodialysis Ayman Abd-Elrahman Mohamed Nsr-Allah ${ }^{a}$, Mohamed Kamar ${ }^{a}$, Ahmed Sharawy ${ }^{a}$, Nermin Raafat ${ }^{b}$
}

${ }^{a}$ Department of Internal Medicine, ${ }^{b}$ Department of Biochemistry and Molecular Biology, Faculty of Medicine, Zagazig University, Zagazig, Egypt

Correspondence to Ayman Abd-Elrahman Mohamed Nsr-Allah, MD, Department of Internal Medicine, Zagazig University, Zagazig, 44519, Egypt. Tel: +20 1005105535 fax: 0020552307830

e-mail: advancedendocrine@hotmail.com

Received 17 April 2018

Accepted 30 July 2018

The Egyptian Journal of Internal Medicine 2018, 30:276-283

\section{Background}

Plasma glucose variability may confer a risk for development of chronic diabetic complications. Glycosylated hemoglobin $(\mathrm{HbA1c})$ reflects average glucose level but not glucose variability, which is measured by mean amplitude of glycemic excursions (MAGEs) and continuous glucose monitoring (CGM).

Aim

To study glucose variability using CGM/MAGE compared with sugar profiles and to assess their value as a therapy guide in patients with diabetic nephropathy on hemodialysis.

\section{Patients and methods}

Group 1 included 50 patients with type 2 diabetes mellitus (T2DM) without diabetic nephropathy. Group 2 included 50 patients with T2DM with diabetic nephropathy. Group 3 included 50 patients with T2DM with diabetic nephropathy on hemodialysis. Measurements of fasting blood glucose, postprandial blood glucose, $\mathrm{HbA1c}$, and glucose variability by MAGE and CGM were done.

\section{Results}

CGM showed significant blood glucose variability (amplitude $>130 \mathrm{mg} / \mathrm{dl}$ in 40 patients $=80 \%$ using CGM and in 45 patients=90\% using MAGE) in dialysis group (group 3) in comparison with glucose variability in nondialysis groups (group 1+group 2) (amplitude $>130 \mathrm{mg} / \mathrm{dl}$ in 20 patients=20\% using either CGM or MAGE). Group 1 showed significant correlations between either CGM or MAGE and all sugar profiles. Group 2 showed significant correlations between CGM and MAGE with either fasting or postprandial blood glucose but not with $\mathrm{HbA1c}$, whereas group 3 showed nonsignificant correlations between either CGM or MAGE and any of sugar profiles.

\section{Conclusion}

CGM/MAGE have high specificity and sensitivity to measure variability of sugar levels, especially in patients with diabetic nephropathy on hemodialysis or not, in which $\mathrm{HbA} 1 \mathrm{c}$ may not be a reliable tool.

\section{Keywords:}

continuous glucose monitoring, diabetic nephropathy, glucose variability, mean amplitude of glycemic excursions

Egypt J Intern Med 30:276-283

(c) 2019 The Egyptian Journal of Internal Medicine 1110-7782

\section{Introduction}

Several interventional and epidemiological studies have confirmed the association between hyperglycemia and the development of diabetic complications in patients with both type 1 and type 2 diabetes [1]. Most of these studies have used glycosylated hemoglobin (HbA1c) as time-averaged mean levels of glycemia [2].

Good blood glucose control improves diabetes-related vascular insult and should be implemented early and maintained. Patients with microvascular complications are particularly prone to atherosclerosis and death [3].

However, research has raised the question that plasma glucose (PG) variability, irrespective of the hyperglycemic levels, can confer more risk for the development of macrovascular and microvascular diabetic complications [4].

There is mounting evidence that increased frequency and magnitude of glycemic variability generates more reactive oxygen species, which result in an increased risk for the development of long-term diabetic complications [5].

Currently, several specific tools have been developed to evaluate blood glucose variability. Among these

This is an open access journal, and articles are distributed under the terms of the Creative Commons Attribution-NonCommercial-ShareAlike 4.0 License, which allows others to remix, tweak, and build upon the work non-commercially, as long as appropriate credit is given and the new creations are licensed under the identical terms. 
measures are mean of daily differences, the mean amplitude of glucose excursions (MAGEs), standard deviation of blood glucose, and meal-related glycemic excursions [6].

Glycemic variability can be measured with different methods including blood glucose values collected monthly, daily, hourly, or every $5 \mathrm{~min}$ [e.g., from continuous glucose monitoring (CGM) data] [7].

This study aimed at assessing glucose variability using CGM/MAGE compared with other blood glucose profiles (fasting blood glucose, postprandial blood glucose, and HbA1c) and at assessing their value as a therapy guide in patients with diabetic nephropathy with or without hemodialysis.

\section{Patients and methods}

The protocol of this study was approved by the local ethics committee in June 2015. All procedures performed were in accordance with the ethical standards of the institutional research committee and with Helsinki declaration and its later amendments. This work was carried out from September 2015 to September 2016 on 150 patients with type 2 diabetic (77 male and 73 females), and their age ranged between 40 and 65 years. Patients older than 65 years, smokers, pregnant women, or patients with malignancies, liver diseases, severe inflammatory or severe concomitant diseases, heart failure, hemoglobin anomalies, and anemia (patients with hemoglobin $<12 \mathrm{~g}$ ) were all excluded. After being informed on the purpose and procedures of the study, all patients signed on an informed consent form and then subdivided into three sex-matched and age matched groups.

Group 1 comprised 50 patients with type 2 diabetes without diabetic nephropathy. There were 24 (48\%) males and 26 (52\%) females, and their ages ranged from 45 to 57 years, with a mean of $49.1 \pm 4.5$ years. Their BMI ranged from 27 to 33 with a mean of 30.3 \pm 1.87 , and their diabetes mellitus (DM) duration ranged from 3 to 6 years, with a mean of $4.4 \pm 0.89$. Their systolic blood pressure (SBP) ranged from 110 to $140 \mathrm{mmHg}$ with a mean of $125 \pm 9.4 \mathrm{mmHg}$, and their diastolic blood pressure (DBP) ranged from 70 to $90 \mathrm{mmHg}$ with a mean of $84 \pm 7.3 \mathrm{mmHg}$. They were on oral anti-DM medications without any antihypertensive medications.

Group 2 comprised 50 patients with type 2 diabetes with diabetic nephropathy. There were 27 (54\%) males and 23 (46\%) females. Their ages ranged from 49 to 59 years, with a mean of $50.1 \pm 4.9$ years. Their BMI ranged from 27 to 34 , with a mean of $30.7 \pm 2.38$, and their DM duration ranged from 5.5 to 14.5 years with a mean of $9.4 \pm 2.8$ years. Their SBP ranged from 110 to $140 \mathrm{mmHg}$, with a mean of 124 $\pm 8.3 \mathrm{mmHg}$, and their DBP ranged from 70 to 95 $\mathrm{mmHg}$, with a mean of $85 \pm 8.3 \mathrm{mmHg}$. A total of 10 patients were on oral anti-DM medication, but 40 patients were on premixed twice daily insulin regimen without antihypertensive medications.

Group 3 comprised 50 patients with type 2 diabetes with diabetic nephropathy on regular hemodialysis three times a week. There were 26 (52\%) males and 24 (48\%) females. Their ages ranged from 50 to 59.5 years, with a mean of $53.1 \pm 4.2$ years, and their BMI ranged from 27 to 35.75 with a mean of $32.6 \pm 2.6$. Their DM duration ranged from 6 to 20 years, with a mean of $11.9 \pm 4.3$ years, and their SBP ranged from 120 to $145 \mathrm{mmHg}$ with a mean of $132.5 \pm 8.5 \mathrm{mmHg}$. Their DBP ranged from 80 to $95 \mathrm{mmHg}$ with a mean of 89 $\pm 5.16 \mathrm{mmHg}$. Overall, 38 patients were on premixed twice daily insulin regimen and 12 patients were on basal bolus insulin regimen without antihypertensive medications.

All patients are subjected to (a) full history taking; (b) full clinical examination, including BMI, which was calculated by dividing weight $(\mathrm{kg})$ by height $\left(\mathrm{m}^{2}\right)$, blood pressure, as well as assessment of patients' files and records (clinical data and investigation) for patients previously diagnosed to have diabetic nephropathy; (c) routine laboratory investigations, including fasting blood glucose, 2-h postprandial blood glucose, HbA1c, complete blood picture, serum uric acid, creatinine, estimated glomerular filtration rate (eGFR), which was calculated by using the Chronic Kidney Disease Epidemiology Collaboration (CKDEPI) equation, which is available at www.nkdep.nih. gov, and the diagnosis of diabetic nephropathy considered according to the National Kidney Foundation classification [8], which is based on both kidney damage [spot urinary albumin/creatinine ratio $(\mathrm{UACR}) \geq 30 \mathrm{mg} / \mathrm{g}]$ and eGFR; (d) determination of glucose variability by two methods: the MAGE technique and CGM.

In the MAGE technique, we used a normal blood glucose meter and recorded 10 readings per day, spaced evenly apart, for 2 days, and then the SD of all the blood glucose measurements taken was calculated. For each glucose reading, we calculated the difference from the previous reading, and recorded this result. If the reading is higher than the previous reading, this was a 
positive change, and if the reading is lower than the previous, this was a negative change. When completed, we had a difference result for every reading except the first reading (as no previous reading to estimate its change). We compared each result calculated against the SD. We ignored the +/- signs and just considered the magnitude of the number. We discarded all numbers where the magnitude of the difference was less than the SD. We calculated the arithmetic mean (average) of the differences that were not eliminated. The mean was calculated by adding all the differences and dividing by the number of data points considered. The calculation of glucose variability was done by the following equation:

$\sum($ The Mean $)=\frac{\lambda}{x}$

$\lambda$ is the absolute value difference between sequential glucose peaks and $x$ is the number of valid observations [9].

In CGM, which is a new measure available using a sensitive but expensive sensor, our patients were subjected to 48-h CGM. The Guardian R REALTime CGMS (Medtronic, Northridge, California, USA) and a Medtronic SOF-SENSOR were used. The system consists of a miniature sensor placed in the subcutaneous fat, a transmitter joined to the sensor, and an external monitor wirelessly communicating with the sensor, with a signal reach of $\sim 2.5 \mathrm{~m}$. The glucose sensing is based on the glucose oxidase method, generating an electrical current. The values are registered every $10 \mathrm{~s}$ and averaged every $5 \mathrm{~min}$. The resulting value is transmitted to the monitor and processed by an algorithm converting the value of glucose concentration in interstitial fluid to the value of PG concentration. This algorithm depends on the calibration value. The monitor displays a fresh calculation of PG every $5 \mathrm{~min}$. The system is capable of reporting $\mathrm{PG}$ concentration in a limited range of $39.6-405 \mathrm{mg} / \mathrm{dl}$, providing up to 288 recordings per day by using Carelink software Medtronic Company Minneapolis, Minnesota (USA) [7].

All data were entered and analyzed using Microsoft excel software. Data were then imported into version 20.0 software for analysis by Microsoft, Redmond, Washington (USA), software for analysis. The qualitative data were represented by the number and percentage of frequencies, and quantitative data were represented by mean \pm SD. Significant differences between frequencies (qualitative variables) in groups were compared by $\chi^{2}$ test. Differences between parametric quantitative independent groups were assessed by $t$ test. Receiver operating characteristic (ROC) curve for cutoff and Kappa agreement to test the agreement were done. $P$ value was set at less than 0.05 for significant results and less than 0.001 for highly significant results.

\section{Result}

Table 1 shows the mean and SD of kidney functions and sugar profile among the studied groups. The dialysis group (group 3 ) has a statistically significant higher level of serum creatinine $(P<0.001)$ in comparison with the other two groups, a higher level of UACR in comparison with the other two groups, and a statistically significant lower level of eGFR $(P<0.001)$ in comparison with the other two diabetic groups. Moreover, the dialysis group (group 3 ) has a statistically significant higher level of fasting blood sugar (FBS) $(P<0.001)$, a statistically significant higher level of postprandial blood sugar (PPBS) $(P<0.001)$, and a statistically significant higher level of HbA1c $(P<0.001)$ in comparison with other groups. Our results in this table also showed that the dialysis group (group 3) has statistically significant higher levels of CGM $(P<0.001)$ in comparison with other two groups, that is, DM (group 1) and diabetic nephropathy (group 2). Table 1 shows that dialysis group (group 3 ) has a statistically significant $(P<0.001)$ higher level of glucose based on MAGE in comparison with the other two groups.

Table 2 shows the effect of hemodialysis on glucose variability. CGM results show more blood glucose variability (variability amplitude $>130 \mathrm{mg} / \mathrm{dl}$ in 40 patients $=80 \%$ ) in dialysis group (group 3) in comparison with nondialysis groups (group 1 and group 2) (variability amplitude $>130 \mathrm{mg} / \mathrm{dl}$ in 20 patients $=20 \%$ ), with a statistically significant difference $(P<0.05)$, and by using MAGE, there was more blood glucose variability (variability amplitude $>130 \mathrm{mg} / \mathrm{dl}$ in 45 patients $=90 \%$ ) in dialysis group (group 3 ) in comparison with nondialysis groups (group 1 and group 2) (variability amplitude $>130 \mathrm{mg} / \mathrm{dl}$ in 20 patients $=20 \%$ ), with a highly statistically significant difference $(P<0.001)$.

Table 3 shows the correlations of MAGE and CGM with other parameters in all studied groups. In DM group (group 1), there were significant positive correlations between MAGE and CGM $(R=0.846$, $P<0.001)$, between MAG and UACR $(R=0.59$, $P<0.05)$, between MAGE and FBS $(R=0.84$, $P<0.001)$, between MAGE and PPBS $(R=0.72$, $P<0.001)$, and between MAGE with HbA1c $(R=0.6, P<0.05)$. Other than that, there were no 
Table 1 Mean and SD of kidney function and sugar profile among the studied groups

\begin{tabular}{|c|c|c|c|c|c|c|c|}
\hline Items & Groups & Mean & SD & Minimum & Maximum & $F$ & $P$ \\
\hline \multirow[t]{3}{*}{ Serum creatinine $(\mathrm{mg} / \mathrm{dl})$} & G1 & 0.97 & 0.113 & 0.80 & 1.10 & 183.0 & $<0.001$ \\
\hline & G2 & 3.2 & 1.28 & 1.90 & 6.20 & & \\
\hline & G3 & 9.2 & 1.142 & 7.40 & 10.50 & & \\
\hline \multirow[t]{3}{*}{ UACR (mg/g) } & G1 & 25.4 & 4.2 & 16.4 & 28.5 & 64.63 & $<0.001$ \\
\hline & G2 & 53.3 & 17.4 & 38.0 & 97.2 & & \\
\hline & G3 & 374.6 & 81.4 & 180.9 & 877.6 & & \\
\hline \multirow[t]{3}{*}{ eGFR $\left(\mathrm{ml} / \mathrm{min} / 1.73 \mathrm{~m}^{2}\right)$} & G1 & 95.2 & 15.82 & 99.5 & 90.3 & 109.81 & $<0.001$ \\
\hline & G2 & 45.9 & 10.04 & 32.10 & 81.10 & & \\
\hline & G3 & 6.1 & 0.74 & 5.10 & 7.20 & & \\
\hline \multirow[t]{3}{*}{ FBS (mg/dl) } & G1 & 131.4 & 12.88 & 115.00 & 155 & 17.84 & $<0.001$ \\
\hline & G2 & 137.3 & 8.83 & 122.00 & 150 & & \\
\hline & $\mathrm{G} 3^{\mathrm{b}}$ & 160.6 & 12.51 & 134.00 & 172 & & \\
\hline \multirow[t]{3}{*}{ PPBS (mg/dl) } & G1 & 164.5 & 15.4 & 145.00 & 200 & 21.66 & $<0.001$ \\
\hline & $\mathrm{G} 2$ & 183 & 21.21 & 155.00 & 210 & & \\
\hline & G3 & 219.9 & 20.34 & 185.00 & 250 & & \\
\hline \multirow[t]{3}{*}{$\mathrm{HbA1c}(\%)$} & G1 & 8.7 & .61 & 7.90 & 9.20 & 133.9 & $<0.001$ \\
\hline & $\mathrm{G} 2$ & 9.4 & .82 & 8.00 & 10.20 & & \\
\hline & G3 & 12.3 & .61 & 10.90 & 12.80 & & \\
\hline \multirow[t]{3}{*}{ CGM (mg/dl) } & G1 & 112.5 & 15.84 & 96.00 & 150 & 13.75 & $<0.001$ \\
\hline & G2 & 148.3 & 22.80 & 120.00 & 200 & & \\
\hline & G3 & 179.4 & 40.91 & 137.00 & 261 & & \\
\hline \multirow[t]{3}{*}{ MAGE (mg/dl) } & G1 & 107.5 & 18.35 & 91.0 & 155 & 28.37 & $<0.001$ \\
\hline & G2 & 149.9 & 13.85 & 130.30 & 170 & & \\
\hline & G3 & 197.8 & 40.35 & 135.50 & 263 & & \\
\hline
\end{tabular}

CGM, continuous glucose monitoring; eGFR, estimated glomerular filtration rate; FBS, fasting blood sugar; HbA1c, glycosylated hemoglobin; MAGE, mean amplitude of glycemic excursion; UACR, urinary albumin/creatinine ratio. Mean and SD of kidney function and sugar profile among the studied groups.

Table 2 Effect of hemodialysis on glucose variability

\begin{tabular}{|c|c|c|c|c|c|c|}
\hline \multirow[t]{2}{*}{ Variability amplitude } & \multicolumn{2}{|c|}{ Groups } & \multirow[t]{2}{*}{ Total } & \multirow[t]{2}{*}{$\chi^{2}$} & \multirow[t]{2}{*}{$P$} & \multirow[t]{2}{*}{ Kappa agreement } \\
\hline & No (G1+G2) & Dialysis & & & & \\
\hline \multicolumn{7}{|l|}{ CGM } \\
\hline$<130[n(\%)]$ & $80(80.0)$ & $10(20.0)$ & $90(60.0)$ & 10 & $<0.05$ & 0.58 \\
\hline$>130[n(\%)]$ & $20(20.0)$ & $40(80.0)$ & $60(40.0)$ & & & \\
\hline \multicolumn{7}{|l|}{ MAGE } \\
\hline$<130[n(\%)]$ & $80(80.0)$ & $5(10.0)$ & 85 (56.7) & 13 & $<0.001$ & 0.78 \\
\hline$>130[n(\%)]$ & $20(20.0)$ & $45(90.0)$ & $65(43.3)$ & & & \\
\hline Total [n (\%)] & $100(100.0)$ & $50(100.0)$ & $150(100.0)$ & & & \\
\hline
\end{tabular}

CGM, continuous glucose monitoring; MAGE, mean amplitude of glycemic excursion.

significant correlations between MAGE and other parameters. Moreover, Table 3 shows significant positive correlations between CGM and UACR $(R=0.64, \quad P<0.05)$, between CGM and FBS $(R=0.658, P<0.05)$, and between CGM and HbA1c ( $R=0.85, P<0.001)$, but there were no significant correlations between CGM and other parameters. In the nephropathy group (group 2), there were significant positive correlations between MAGE and UACR $(R=0.63, P<0.001)$, FBS $(R=0.55, P<0.05)$, and PPBS $(R=0.77, P<0.001)$, but no significant correlation between MAGE and other parameters. Moreover, this table shows significant positive correlations between CGM and UACR $(R=0.88$, $P<0.001)$, FBS $(R=0.79, \quad P<0.001)$, and PPBS
$(R=0.64, P<0.05)$. Other than that, no significant correlation was found between CGM and other parameters. In dialysis group (group 3), there were significant positive correlations between MAGE and CGM $\quad(R=0.895, \quad P<0.001), \quad$ dialysis duration $(R=0.785, \quad P<0.001), \quad$ and $\quad$ UACR $\quad(R=0.81$, $P<0.001)$. Other than that, no significant correlation was found between MAGE and other parameters. Moreover, this table shows significant positive correlations between CGM and dialysis duration $(R=0.822, P<0.001)$ and UACR $(R=0.78$, $P<0.001$ ), but no significant correlation between CGM and other parameters Table 4) shows the ROC curve for MAGE and CGM in nephropathy group (group 2) and hemodialysis group (group 3). In 
Table 3 Correlations of mean amplitude of glycemic excursion and continuous glucose monitoring with renal functions and sugar profile

\begin{tabular}{|c|c|c|c|c|}
\hline Groups & Parameters & & MAGE & CGM \\
\hline \multirow[t]{12}{*}{ G1 } & MAGE/CGM & $R$ & \multicolumn{2}{|c|}{$0.846<0.001$} \\
\hline & & $P$ & & \\
\hline & \multirow[t]{2}{*}{ Serum creatinine $(\mathrm{mg} / \mathrm{dl})$} & $R$ & 0.121 & 0.452 \\
\hline & & $P$ & 0.739 & 0.190 \\
\hline & \multirow[t]{2}{*}{ UACR (mg/g) } & $R$ & 0.59 & 0.64 \\
\hline & & $P$ & $<0.05$ & $<0.05$ \\
\hline & \multirow[t]{2}{*}{ FBS $(\mathrm{mg} / \mathrm{dl})$} & $R$ & 0.84 & 0.65 \\
\hline & & $P$ & $<0.001$ & $<0.05$ \\
\hline & \multirow[t]{2}{*}{ PPBS (mg/dl) } & $R$ & 0.72 & 0.69 \\
\hline & & $P$ & $<0.001$ & 0.060 \\
\hline & \multirow[t]{2}{*}{$\mathrm{HbA1c}(\%)$} & $R$ & 0.60 & 0.855 \\
\hline & & $P$ & $<0.001$ & 0.060 \\
\hline \multirow[t]{12}{*}{ G2 } & \multirow[t]{2}{*}{ MAGE/CGM } & $R$ & \multirow{2}{*}{\multicolumn{2}{|c|}{$-0.4270 .219$}} \\
\hline & & $P$ & & \\
\hline & \multirow[t]{2}{*}{ Serum creatinine $(\mathrm{mg} / \mathrm{dl})$} & $R$ & -0.003 & 0.203 \\
\hline & & $P$ & 0.994 & 0.574 \\
\hline & \multirow[t]{2}{*}{ UACR (mg/g) } & $R$ & 0.63 & 0.88 \\
\hline & & $P$ & $<0.001$ & $<0.001$ \\
\hline & \multirow[t]{2}{*}{ FBS (mg/dl) } & $R$ & 0.55 & 0.79 \\
\hline & & $P$ & $<0.05$ & $<0.001$ \\
\hline & \multirow[t]{2}{*}{ PPBS (mg/dl) } & $R$ & 0.77 & 0.646 \\
\hline & & $P$ & $<0.001$ & $<0.05$ \\
\hline & \multirow[t]{2}{*}{$\mathrm{HbA1c}(\%)$} & $R$ & 0.32 & 0.41 \\
\hline & & $P$ & 0.081 & 0.072 \\
\hline \multirow[t]{14}{*}{ G3 } & \multirow[t]{2}{*}{ MAGE/CGM } & $R$ & \multicolumn{2}{|c|}{$0.895<0.001$} \\
\hline & & $P$ & & \\
\hline & \multirow[t]{2}{*}{ Dialysis duration } & $R$ & 0.785 & 0.822 \\
\hline & & $P$ & $<0.001$ & $<0.001$ \\
\hline & \multirow[t]{2}{*}{ Serum creatinine $(\mathrm{mg} / \mathrm{dl})$} & $R$ & 0.241 & 0.316 \\
\hline & & $P$ & 0.503 & 0.373 \\
\hline & \multirow[t]{2}{*}{ UACR $(\mathrm{mg} / \mathrm{g})$} & $R$ & 0.81 & 0.78 \\
\hline & & $P$ & $<0.001$ & $<0.001$ \\
\hline & \multirow[t]{2}{*}{ FBS (mg/dl) } & $R$ & -0.290 & -0.328 \\
\hline & & $P$ & 0.416 & 0.355 \\
\hline & \multirow[t]{2}{*}{ PPBS (mg/dl) } & $R$ & -0.224 & -0.268 \\
\hline & & $P$ & 0.535 & 0.454 \\
\hline & \multirow[t]{2}{*}{$\mathrm{HbA1c}(\%)$} & $R$ & -0.279 & -0.444 \\
\hline & & $P$ & 0.435 & 0.199 \\
\hline
\end{tabular}

CGM, continuousglucose monitoring; FBS, fasting blood sugar; $\mathrm{HbA1c}$, glycosylated hemoglobin; MAGE, mean amplitude of glycemic excursion; PPBS, postprandial blood sugar; UACR, urinary albumin/creatinine ratio. Correlations of mean amplitude of glycemic excursion and continuous glucose monitoring with renalfunctions and sugar profile. nephropathy group, MAGE at a cutoff for nephropathy of more than 116.7 had a sensitivity of $90.0 \%$ and specificity $90.0 \%$ in detecting glucose variability, and CGM at a cutoff of more than 106.5 had sensitivity of $90.0 \%$ and specificity of $90.0 \%$. In hemodialysis group (group 3), MAGE at a cutoff for hemodialysis of more than 136.5 had a sensitivity of $90.0 \%$ and specificity of $80.0 \%$ in detecting glucose variability and CGM at a cutoff of more than 131 had a sensitivity of $80.0 \%$ and specificity of $80.0 \%$ in detecting glucose variability.

\section{Discussion}

In our study, there is a statistically significant increase of FBS and PPBG in group 3 more than the other two groups (group 1 and group 2). This result is in agreement with Abe et al. [10] whose stated that hemodialysis can lead to hyperglycemia by decreased levels of plasma immune reactive insulin, even that hemodialysis sessions can decrease PG level if measured before or after the hemodialysis sessions.

In this work, we found a statistically significant increase of $\mathrm{HbA} 1 \mathrm{c}$ in group 3 more than the other two groups. This is in agreement with Jin et al. [11] whose stated that diabetic hemodialysis patients are associated with bad diabetes control with $\mathrm{HbA1c}$ more than 7\%, which is common in uremic states, as the uremic toxin influences glucose homeostasis by decreasing insulin sensitivity, increasing hepatic gluconeogenesis, and decreasing cellular glucose utilization in end-stage renal disease. However, Hoshino et al. [12] reported that simple cutoff values for $\mathrm{HbA1c}$ will be undependable by variable $\mathrm{RBC}$ s half-life or different hemoglobin glaciation as there are different blood $\mathrm{pH}$ values, and $\mathrm{Wu}$ et al. [13], mentioned that there is no correlation of the level of HbA1c and the mean of blood glucose values between diabetic patients with healthy renal function and patients with CKD without hemodialysis.

Furthermore, HbA1c in diabetic patients on hemodialysis and patients with CKD without

Table 4 Receiver operating characteristic curve for mean amplitude of glycemic excursion and continuous glucose monitoring in both nephropathy groups (G2 and G3)

\begin{tabular}{|c|c|c|c|c|c|c|c|c|}
\hline \multirow[t]{2}{*}{ Groups } & \multirow[t]{2}{*}{ MAGE/CGM } & \multirow[t]{2}{*}{ Area } & \multirow[t]{2}{*}{ Cutoff } & \multirow[t]{2}{*}{$P$} & \multicolumn{2}{|c|}{$95 \%$ confidence interval } & \multicolumn{2}{|c|}{ Validity } \\
\hline & & & & & Lower bound & Upper bound & Lower bound (\%) & Upper bound (\%) \\
\hline \multicolumn{9}{|l|}{ G2 } \\
\hline & MAGE & 0.540 & $>116.7$ & 0.025 & 0.331 & 0.749 & 90.0 & 90.0 \\
\hline & CGM & 0.577 & $>106.5$ & 0.015 & 0.375 & 0.780 & 90.0 & 90.0 \\
\hline \multirow[t]{2}{*}{ G3 } & MAGE & 0.930 & $>136.5$ & 0.000 & 0.825 & 1.000 & 90.0 & 80.0 \\
\hline & CGM & 0.880 & $>131$ & 0.001 & 0.755 & 1.000 & 80.0 & 80.0 \\
\hline
\end{tabular}

CGM, continuous glucose monitoring; MAGE, mean amplitude of glycemic excursion. 
hemodialysis will be lower than HbA1c in type 2 diabetic patients with healthy kidney function owing to variable carbohydrate metabolism in patients on dialysis leading to more susceptibility to hypoglycemia, and owing to the fact that the interaction time between hemoglobin and blood glucose is shorter in patients with CKD, which leads to decrease hemoglobin glycosylation, in addition to that anemia is considered as a direct cause of lesser correlation [14].

Our study showed a statistically significant increase of mean blood glucose level by using CGM and MAGE in group 3 more than other two groups (group 1-group 2 ), and there was a statistically significant increase of mean blood glucose level by using CGM and MAGE in group 2 more than group 1 . This coincides with Massimo et al. [15] who mentioned that glucose levels measured by MAGE in patients with type 2 diabetes mellitus (T2DM) on hemodialysis are more variable than type 2 diabetic patients not on hemodialysis, as there is decrease in glucose levels during hemodialysis session than increase of blood glucose levels after the session, and this variability phenomena is evident in patients with bad DM control. Moreover, this result is in concordance with study of Lailis et al. [16] who considered CGM and MAGE as an advanced option in providing real information about the current levels of blood glucose and providing an actual feedback about the effect of antidiabetic drugs, especially insulin administration, and it gives alarm in dangerous extremes of blood glucose levels either severe hyper glycemia or hypoglycemia. In addition, MAGE is considered as an excellent solution for calculation of the glycemic variability, and it also gives a direct relation with $\mathrm{HbA} 1 \mathrm{c}$ and postprandial hyperglycemia in diabetic patients [17].

In this study, there was a significant correlation between MAGE or CGM with glucose profile (FBG, PPBG, and HbA1c) in DM group (group 1) and in diabetic nephropathy group (group 2), but no significant correlation was found between either MAGE or CGM and glucose profile (FBG, PPBG, and $\mathrm{HbA1c}$ ) in hemodialysis group (group 3). These results are concordant with Nalysnyk et al. [18] who stated that the changes in the blood glucose levels through time represent glucose variability and can be measured precisely by using MAGE or CGM. Glucose variability reflects the changes in the levels of blood glucose throughout $24 \mathrm{~h}$, with good correlation between MAGE or CGM and this variability in both fasting and postprandial blood glucose values. Moreover, another study revealed that in diabetic patients, the postprandial hyperglycemia leads to glucose variability [19].

On the contrary, the patients with same levels of $\mathrm{HbA1c}$ can have variable daily sugar readings with different excursions both in its numbers and durations in T2DM patients with nephropathy, especially if on hemodialysis [20]. However MAGE and CGM are not always represented by $\mathrm{HbA} 1 \mathrm{c}$ because $\mathrm{HbA} 1 \mathrm{c}$ is the average of blood glucose levels but cannot measure glucose variability [17].

Our results showed a significant positive correlation between MAGE or CGM with UACR in all our three groups, and this result is in concordance with study of Nalysnyk et al. [18] who stated that PG variability, irrespective of the hyperglycemic levels, can confer mored risk for the development of macrovascular and microvascular diabetic complications and can be explained by increasing frequency and magnitude of glycemic variability, which generates more reactive oxygen species and results in an increased risk for the development of long-term diabetic complications [5].

Our results showed more blood glucose variability in dialysis group (group 3) in comparison with glucose variability in nondialysis groups (group 1 and group 2). Variability in blood glucose levels in patients with $\mathrm{CKD}$ is owing to spontaneous hypoglycemia by chronic uremia and also attributed to impaired gluconeogenesis owing to deficient alanine (precursors of gluconeogenesis) with impaired renal insulin degradation and clearance. Dialysis also predisposes to hypoglycemia, which is possibly because of the chronic state of malnutrition. Postdialysis hypoglycemia is secondary to glucose-induced hyperinsulinemia, which is caused by the high glucose content in the dialysate. Moreover, another hypothesis is that increased glucose variability in patients with chronic renal disease reflects the resistance to insulin, either endogenous or exogenous, leading to hyperglycemia and more glycemic fluctuations. Insulin resistance in CKD may be a consequence of chronic inflammation, vitamin $\mathrm{D}$ deficiency, excess visceral fat, oxidative stress, acidosis, decreased physical activity, anemia, or other factors [21]. Moreover, $\mathrm{Xu}$ et al. [22] mentioned that glucose variability parameters are correlated with diabetic nephropathy in T2DM with wide glucose excursions by deteriorating the kidney function.

In our study, we found in nephropathy group, MAGE at a cutoff for nephropathy of more than 116.7 had a sensitivity of $90.0 \%$ and specificity of $90.0 \%$, whereas CGM at a cutoff of more than 106.5 had a sensitivity of 
$90.0 \%$ and specificity of $90.0 \%$ in detecting glucose variability. Moreover, we found in dialysis group that MAGE at a cutoff for dialysis of more than 136.5 had a sensitivity of $90.0 \%$ and specificity of $80.0 \%$ whereas CGM at a cutoff of more than 131 had a sensitivity of $80.0 \%$ and specificity $80.0 \%$ in detecting glucose variability.

Cichosz et al. [23] investigated either the algorithm of autonomic modulation based on heart rate variability (HRV) or data based on CGM device was suitable for sugar readings and prediction of hypoglycemia and for improvement of hypoglycemic detection during normal daily activities. A total of 12 hypoglycemic attacks (glucose levels $<70 \mathrm{mg} / \mathrm{dl}$ ) and 237 normoglycemic levels were included. For HRV model, ROC area under the curve (AUC) of 0.69 had a high sensitivity of $100 \%$ and the specificity was $69 \%$; for the CGM model, ROC AUC of 0.92 had a corresponding sensitivity of $100 \%$ and specificity of $71 \%$, and for the CGM plus HRV model, ROC AUC of 0.96 had a sensitivity of $100 \%$ and specificity of $91 \%$. Daniel et al. [24] stated that there is feasibility and efficacy of changing systematic Self monitoring of blood glucose (SMBG) by CGM to increase quantitative and qualitative feedback. By CGM device, diabetic patients can know their current blood glucose and its fluctuations and rate of any extreme readings. Finally our study revealed that CGM has a distinguished pattern of glucose variability in diabetic patients specially in nephropathy and in hemodialysis patients, which is in agreement with a former study by Le Floch and Kessler [25].

\section{Conclusion}

We can conclude that glucose variability is present in diabetic patients with diabetic nephropathy especially on hemodialysis, and this variability may be related to diabetic complication. HbA1c is a tool for glycemic monitoring; however, in patients with diabetic nephropathy and especially those who are on hemodialysis, it may not be a reliable tool. CGM/ MAGE have high specificity and sensitivity in measuring variability of sugar level in diabetic patients with diabetic nephropathy with or without hemodialysis.

We recommend the control of blood sugar in patients with diabetic nephropathy on hemodialysis or not by a flexible and intensive regimen using insulin in a basal bolus manner to avoid any variability-related morbidity and mortality.

\section{Acknowledgements}

Ayman Abd-Elrahman designed the research and collected the clinical data. Nermin Raafat supervised the laboratory methods. Ahmed Sharawy performed statistical analysis and Mohamed Kamar wrote the paper.

\section{Financial support and sponsorship \\ Nil.}

\section{Conflicts of interest}

There are no conflicts of interest.

\section{References}

1 American Diabetes Association. Microvascular complications and foot care. Sec. 9 in Standards of Medical Care in Diabetes 2016. Diabetes Care 2016; 39 (Suppl. 1):S72-S80.

2 Cavalot F. Glycemic variability and vascular complications of diabetes. Diabetes Obese Metab 2013; 1 (Suppl. 2):3-8

3 Chawla A, Chawla RS. Microvascular and macrovascular complications in diabetes mellitus: distinct or continuum? Indian J Endocrinol Metab 2016; 20:546-551.

4 Jacobson AM, Braffett BH, Cleary PA, Gubitosi-Klug RA, Larkin ME. The long-term effects of type 1 diabetes treatment and complications on healthrelated quality of life: a 23-year follow-up of the Diabetes Control and Complications/Epidemiology of Diabetes Interventions and Complications cohort. Diabetes Care 2013; 36:3131-3138

5 Kramer CK, Choi H, Zinman B, Retnakaran R. Glycemic variability in patients with early type 2 diabetes: the impact of improvement in betacell function. Diabetes Care 2014; 37:1116-1123.

6 Tay J, Thompson $\mathrm{CH}$, Brinkworth GD. Glycemic variability: assessing glycemia differently and the implications for dietary management of diabetes. Annu Rev Nutr 2015; 35:389-424.

7 Prazny M, Soupal J. Glycemic variability and continuous monitoring of glycemia. Vnitr Lek 2014; 60:757-763.

8 Ketteler M, Block GA, Evenepoel P, Fukagawa M, Herzog CA, McCann L, et al. Executive summary of the $2017 \mathrm{KDIGO}$ chronic kidney disease-mineral and bone disorder (CKD-MBD) guideline update. Kidney Int 2017; 92:26-36.

9 Kovatchev BP, Patek SD, Ortiz EA, Breton MD. Assessing sensor accuracy for non-adjunct use of continuous glucose monitoring. Diabetes Technol Ther 2015; 17:177-186.

10 Abe M, Kaizu K, Matsumoto K. Evaluation of the hemodialysis-induced changes in plasma glucose and insulin concentrations in diabetic patients: comparison between the hemodialysis and non-hemodialysis days. Ther Apher Dial 2014; 11:288-295.

11 Jin YP, Su XF, Yin GP, Xu XH, Lou JZ, Chen JJ, et al. Blood glucose fluctuations in hemodialysis patients with end stage diabetic nephropathy. $J$ Diabetes Complications 2014; 29:395-399.

12 Hoshino J, Molnar MZ, Yamagata K, Ubara Y, Takaichi K, Kovesdy CP, Kalantar-Zadeh K. Developing an $\mathrm{HbA}$ (1c)-based equation to estimate blood glucose in maintenance hemodialysis patients. Diabetes Care 2013; 36:922-927

13 Wu TE, Chen YS, Chen HS. Effects of glucose and blood pressure control on diabetic kidney disease in old patients with type 2 diabetes. Diabetol Metab Syndr 2014; 6:81.

14 Coelho $S$. What is the role of hba1c in diabetic hemodialysis patients? Semin Dial 2006; 29:19-23.

15 Massimo G, Ilenia M, Sergio D, Vincenzo C, Gianluca L, Fabrizio F, et al. Glycemic pattern in diabetic patients on hemodialysis: continuous glucose monitoring (CGM) analysis. Blood Purif 2014; 38:68-73.

16 Lailis S, Askander T, Indra M, Sargowo D, Muladi L. Expert system for blood glucose fluctuations measurement based on MAGE and $\mathrm{HbA1}$ c on diabetic using K-NN (nearest neighbor). J Basic Appl Sci Res 2014; 4:135-141.

17 Siegelaar SE, Holleman F, Hoekstra JB, DeVries JH. Glucose variability; does it matter? Endocr Rev 2012; 31:171-182.

18 Nalysnyk L, Hernandez-Medina M, Krishnarajah G. Glycaemic variability and complications in patients with diabetes mellitus: evidence from a systematic review of the literature. Diabetes Obes Metab 2012; 12:288-298.

19 Hsu CR, Chen YT, Sheu WH. Glycemic variability and diabetes retinopathy: a missing link. J Diabetes Complications 2015; 29:302-306. 
20 Riveline JP, Teynie J, Belmouaz S, Franc S, Dardari D, Bauwens M. Glycaemic control in type 2 diabetic patients on chronic haemodialysis: use of a continuous glucose monitoring system. Nephrol Dial Transplant 2009; 24:2866-2871.

21 Zammitt NN, Frier BM. Hypoglycaemia in type 2 diabetes and in elderly. John Wiley \& Sons Ltd 2014:230-262.

22 Xu F, Zhao L-H, Su J-B, Chen T, Wang X-Q, Chen J-F, et al. The relationship between glycemic variability and diabetic peripheral neuropathy in type 2 diabetes with well-controlled HbA1c. Diabetol Metab Syndr 2014; 6:355-367.
23 Cichosz SL, Frystyk J, Tarnow L. Combining information of autonomic modulation and CGM measurements enables prediction and improves detection of spontaneous hypoglycemic events. J Diabetes Sci Technol 2015; 9:132-137.

24 Daniel J, Ann G, Moncrief M, Diamond A, William S, Hegde S, Anthony L. Continuous glucose monitoring in the self-management of type 2 diabetes: a paradigm shift. Diabetes Care 2016; 39:e71-e73.

25 Le Floch JP, Kessler L. Glucose variability: comparison of different indices during continuous glucose monitoring in diabetic patients. J Diabetes Sci Technol 2016; 10:885-891. 